\title{
Recombinant human antithrombin III restores heparin responsiveness and decreases activation of coagulation in heparin-resistant patients during cardiopulmonary bypass
}

\author{
M. S. Avidan, MD, ${ }^{a}$ J. H. Levy, MD, ${ }^{b}$ H. van Aken, $M D,{ }^{c}$ R. O. Feneck, MD, ${ }^{d}$ R. D. Latimer, MD, ${ }^{e}$ E. Ott, $M D,{ }^{f}$ \\ E. Martin, MD, ${ }^{g}$ D. E. Birnbaum, MD, ${ }^{h}$ L. J. Bonfiglio, BSN, CRNA, ${ }^{,}$D. K. Kajdasz, PhD, ${ }^{j}$ and G. J. Despotis, MD ${ }^{k}$
}

\begin{abstract}
From the Departments of Anesthesiology and Cardiothoracic Surgery ${ }^{\mathrm{a}}$ and the Departments of Anesthesiology, Surgery, Pathology, and Immunology, ${ }^{\mathrm{k}}$ Washington University School of Medicine, St Louis, Mo; the Department of Anesthesiology, Emory University School of Medicine, and the Division of Cardiothoracic Anesthesiology and Critical Care, Emory Healthcare, ${ }^{\text {b }}$ Atlanta, Ga; the Department of Anesthesiology, ${ }^{c}$ Westfalische Wilhelms-Universiteit, Münster, Klinik für Anasthesiologie, Münster, Germany; the Department of Anaesthesia, ${ }^{\mathrm{d}}$ St Thomas' Hospital, London, United Kingdom; the Department of Anaesthesia, ${ }^{\mathrm{e}}$ Papworth Hospital, Cambridge, United Kingdom; the Department of Anesthesiology, ${ }^{\mathrm{f}}$ Universitäts-Klinikum Großhadern, Institut für Anästhesiologie, Munich, Germany; the Department of Anesthesiology, ${ }^{\mathrm{g}} \mathrm{Ru}-$ precht-Karls-Universiteit, Heidelberg, Germany; the Department of Anesthesiology, ${ }^{\mathrm{h}}$ Klinikum der Universiteit Regensburg, Regensburg, Germany; Clinical Research, ${ }^{\mathrm{i}}$ GTC Biotherapeutics, Inc, Framingham, Mass; and Eli Lilly \& Co, ${ }^{j}$ Lilly Corporate Center, Indianapolis, Ind.
\end{abstract}

Supported in part by a research grant from GTC Biotherapeutics, Inc. Drs Levy and Despotis have received grant support from GTC Biotherapeutics, Inc, and have been consultants on GTC Biotherapeutics, Inc, Medical Advisory Boards. Judy Bonfiglio is employed by GTC Biotherapeutics.

Received for publication Aug 2, 2004; revisions received Sept 26, 2004; accepted for publication Oct 6, 2004.

Address for reprints: M. S. Avidan, MBBCh, Department of Anesthesiology, Box 8054, Washington University School of Medicine, 660 South Euclid, St Louis, MO 63110 (E-mail: avidanm@msnotes. wustl.edu).

J Thorac Cardiovasc Surg 2005;130:107-13 $0022-5223 / \$ 30.00$

Copyright (C) 2005 by The American Association for Thoracic Surgery

doi:10.1016/j.jtcvs.2004.10.045
Objectives: We sought to evaluate the efficacy of recombinant human antithrombin III for restoration of heparin responsiveness in heparin-resistant patients scheduled for cardiac surgery.

Methods: This was a multicenter, randomized, double-blind, placebo-controlled study in heparin-resistant patients undergoing elective cardiac surgery. Patients were considered heparin resistant if the activated clotting time was less than 480 seconds after $400 \mathrm{U} / \mathrm{kg}$ heparin. Fifty-two heparin-resistant patients were randomized into 2 cohorts. One cohort received a single bolus $(75 \mathrm{U} / \mathrm{kg})$ of recombinant human antithrombin III $(\mathrm{n}=28)$, and the other, the placebo group $(\mathrm{n}=24)$, received a normal saline bolus. If the activated clotting time remained less than 480 seconds, this was defined as treatment failure, and 2 units of fresh frozen plasma were transfused. Patients were monitored for adverse events during hospitalization.

Results: Six (21\%) of the patients in the recombinant human antithrombin III group received fresh frozen plasma transfusions compared with 22 (92\%) of the placebotreated patients $(P<.001)$. Two units of fresh frozen plasma did not restore heparin responsiveness. There was no increased incidence of adverse events associated with recombinant human antithrombin III administration. Postoperative 24-hour chest tube bleeding was not different in the 2 groups. Surrogate measures of hemostatic activation suggested that there was less activation of the hemostatic system during cardiopulmonary bypass in the recombinant human antithrombin III group.

Conclusion: Treatment with recombinant human antithrombin III in a dose of 75 $\mathrm{U} / \mathrm{kg}$ is effective in restoring heparin responsiveness and promoting therapeutic anticoagulation for cardiopulmonary bypass in the majority of heparin-resistant patients. Two units of fresh frozen plasma were insufficient to restore heparin responsiveness. There was no apparent increase in bleeding associated with recombinant human antithrombin III.

$\mathrm{E}$ ffective anticoagulation is a prerequisite for the safe institution of cardiopulmonary bypass (CPB). By inducing a conformational change in the antithrombin III (ATIII) tertiary structure, unfractionated heparin increases the affinity of ATIII for thrombin approximately 1000 -fold. ATIII is a naturally occurring plasma protein that inhibits thrombin and factor $\mathrm{Xa}$ (and other circulating coagulation factors) and binds to heparan sulfate moieties on the vascular endothelium to help maintain homeostasis of the hemostatic system. Heparin's anticoagulant effect is closely tied to ATIII activity. Accordingly, abnormally low ATIII plasma activity might lead to an altered anticoagulant response to heparin. During $\mathrm{CPB}$, therapeutic anticoagulation is usually monitored by the activated clotting time (ACT), a simple and rapid point-of-care test. The decision to initiate CPB after heparin administration hinges on attaining an adequate ACT. Limited data exist that 
define the optimal ACT for initiation of $\mathrm{CPB},{ }^{1}$ but historically, values of less than 300 seconds were associated with grossly visible clots in the bypass circuit. In a 1000-member survey of the Society of Cardiovascular Anesthesiology and American Society of Extracorporeal Circulation, the target ACT used by $82 \%$ of responders was 400 to 480 seconds or longer, with an additional $4.5 \%$ targeting an even higher $\mathrm{ACT}^{2}$

Heparin resistance can be defined as the failure to achieve the desired ACT after a standard dose of heparin (300-400 U/kg). ${ }^{2}$ Failure to achieve an acceptable ACT for CPB is usually managed by additional heparin administration. Less frequently, fresh frozen plasma (FFP) is administered in an attempt to restore heparin responsiveness. Investigators have found the incidence of heparin resistance among patients undergoing cardiac surgery to vary between $4 \%$ and $13 \%$, depending on the target ACT and heparin dose required and on whether patients have recently received heparin. $^{3,4}$

Lower ATIII levels are associated with a decreased heparin dose response (HDR), as measured by the ACT. ${ }^{5}$ ATIII supplementation has been used to improve the ACT response to heparin ${ }^{6}$ and is associated with better thrombin inhibition. ${ }^{5}$ Initial studies suggest that recombinant human ATIII (rhATIII) is effective in restoring heparin responsiveness in patients with heparin resistance. ${ }^{7}$ A previous study established that single dosing with $75 \mathrm{U} / \mathrm{kg}$ or more of rhATIII resulted in plasma ATIII levels approximating $100 \%$ activity that were maintained throughout $\mathrm{CPB} .^{7}$

FFP is readily available at most institutions and has been effectively used as an exogenous source of ATIII to restore heparin responsiveness. ${ }^{8}$ FFP as a source of ATIII is one of the medically accepted treatments for heparin resistance. FFP, however, is not an innocuous intervention and carries the risk of several complications, including viral infections and allergic reactions. It is also possible that FFP increases ATIII concentrations only when large volumes ( $>2 \mathrm{~L}$ ) are administered. The current study was therefore designed to assess the efficacy and safety of rhATIII for improving heparin responsiveness in heparin-resistant patients undergoing elective cardiac surgery involving $\mathrm{CPB}$, thereby avoiding the need for FFP. The trial also aimed to assess whether rhATIII at a dose of $75 \mathrm{U} / \mathrm{kg}$ increases plasma ATIII activity and inhibits thrombin and fibrinolytic activity more effectively than 2 units of FFP.

\section{Methods}

Review and written approval of the study protocol by the independent ethics committees or institutional review boards was obtained before initiation of each study site. Written informed consent was obtained from each enrolled participant.

\section{Study Design}

This multicenter, randomized, double-blind, placebo-controlled rhATIII efficacy study included patients at 6 European study centers undergoing first-time elective coronary artery bypass surgery requiring CPB. This population was chosen because FFP is sometimes administered as a source of ATIII to facilitate heparin anticoagulation in heparin-resistant patients before or during CPB. ${ }^{8}$

Patients 18 to 85 years of age scheduled for elective cardiac surgery and requiring $\mathrm{CPB}$ were included in the study. No patients received aprotinin or any other antifibrinolytic agent. The bypass circuits were not heparin coated, systems were open, cardiotomy suction was used, and patients were cooled to $32^{\circ} \mathrm{C}$. Patients who had a baseline HDR slope of 80 seconds or less with the Hepcon Hemostasis Management System (Medtronics, Minneapolis, Minn) were eligible. In addition, patients who had received intravenous heparin before surgical intervention, regardless of the HDR slope, were also eligible. Patients were excluded who had recently received or were receiving one or more of the following medications: warfarin (within 3 days); streptokinase; tissue plasminogen activator; abciximab, eptifibatide, or tirofiban; or clopidogrel. Also excluded were patients with preexisting coagulopathy defined as a history of bleeding problems or a laboratory history of a bleeding disorder.

Patients were considered heparin resistant if their ACT was less than 480 seconds (ACT II, Medtronics) 5 minutes after receiving a heparin dose of $300 \mathrm{U} / \mathrm{kg}$, followed by an additional $100 \mathrm{U} / \mathrm{kg}$ (total heparin dose, $400 \mathrm{U} / \mathrm{kg}$ ). Heparin-resistant patients were randomized into one of the 2 treatment groups. One group received a single-bolus $75 \mathrm{U} / \mathrm{kg}$ intravenous injection of rhATIII, and the other received a single-bolus intravenous injection of a normal saline placebo. If after the randomized bolus the ACT remained less than 480 seconds, this was defined as treatment failure, and 2 units of FFP were transfused. Additional heparin was administered during CPB as guided by the automated protamine titration method (Hepcon, Medtronics) and ACT values. Reversal of heparin with protamine after CPB was per institutional protocol.

Blood for measurement of hematologic parameters was obtained just before study drug administration ( 0 minutes), at 30 minutes after the initiation of $\mathrm{CPB}$, and just before heparin reversal with protamine. These parameters included Kaolin ACT without heparinase and plasma ATIII activity levels. The ACT II (Automated Coagulation Timer, Medtronics) device was used for measuring the ACT during the treatment period. ACTrac (Medtronics), an electronic calibration device, was used in conjunction with the ACT II device to insure that evaluation values by using Hepcon (Medtronics), and study values with ACT II were consistent between the 2 devices. Additional blood was obtained at baseline and 4 weeks postoperatively to monitor serum samples for antibody formation to rhATIII.

All adverse events occurring from the time of study drug administration until hospital discharge and during the 4-week postoperative follow-up period were recorded. Blood and blood components transfused in the perioperative period were recorded. Postoperative blood loss through chest tube drainage was also recorded. Four weeks postoperatively, a blood sample for rhATIII was obtained, and a postoperative review for serious adverse events was performed. 
The study's primary efficacy end point was whether rhATIII restores heparin responsiveness. Patients who met the requirement for FFP but did not receive FFP were included in the analysis of patients who received FFP (ie, intention to treat). Secondary end points were comparisons between study groups of ACT values and plasma ATIII activity levels in the peri-CPB period. Other secondary evaluations included the inhibition of thrombin activity, as measured by changes in levels of fibrin monomer, the inhibition of fibrinolysis as measured by changes in D-dimer levels, and the changes in plasma ATIII activity levels at 2 time points: 30 minutes after initiation of $\mathrm{CPB}$ and before protamine administration.

\section{Study Medications}

RhATIII, manufactured by GTC Biotherapeutics (Framingham, Mass), was supplied in clear, glass, single-dose $20-\mathrm{mL}$ vials and was refrigerated at $2^{\circ} \mathrm{C}$ to $8^{\circ} \mathrm{C}$ until reconstitution. The institutional research pharmacist at each study site prepared all study materials and was the only person at the site who knew the treatment assignment. Normal saline, indistinguishable from the reconstituted rhATIII at the designated concentration, served as the placebo control.

\section{Statistics}

It was anticipated that up to 500 patients would have to be screened and consented to identify 52 eligible patients. With 24 patients in each group, there would be $80 \%$ power to detect a $40 \%$ absolute reduction $(65 \%$ vs $25 \%)$ in the proportion of patients requiring FFP. A total of 52 randomized patients, 26 in each treatment group, was recommended for the trial to account for a dropout rate of approximately $10 \%$. The primary end point of the study was the comparison of the proportion of patients in each treatment group requiring the infusion of FFP after administration of study medication but before initiation of CPB.

The primary efficacy analysis, the difference between the proportions of patients requiring FFP in each treatment group, was assessed by using the Pearson $\chi^{2}$ test or the Barnard unconditional exact test, depending on the data distribution. Differences in the treatment effect on the odds of requiring FFP were assessed by using logistic regression. Odds ratios comparing treatment groups and $95 \%$ confidence intervals were calculated if indicated.

The secondary efficacy end points, ACT values, and plasma AT activity, were examined at 2 time points: 30 minutes after initiation of CPB and before protamine administration. Differences between the group means were assessed at each time point by analysis of variance. Cohort differences in change from baseline (time 0) in levels of fibrin monomer, D-dimer, and plasma ATIII at 30 minutes after CPB initiation and before protamine were assessed with an analysis of variance model or rank sum test, depending on data distribution. Two additional parameters, indicators of thrombin activation that were recognized as useful but were evaluated after completion of the study, were measured at baseline (time 0), at 30 minutes after initiation of $\mathrm{CPB}$, and at the preprotamine time point in retained plasma samples. These included prothrombin fragment 1.2 and thrombin-antithrombin complex. Although not prospectively defined, evaluation of these parameters was performed under blinded conditions.
The significance of shifts in safety parameters was evaluated within each group by the McNemar test for binary variables and the generalized McNemar test for more than 2 categories. The difference between proportions of patients experiencing other safety-related events (ie, rehospitalization, incidence of red blood cell transfusion, platelet transfusion, additional blood product transfusion, duration of surgical intensive care unit stay, duration of hospitalization, 30-day incidence of rehospitalization, myocardial infarction, stroke, or death) were assessed by the Barnard unconditional exact test.

\section{Results}

Across all of the study centers, the distribution of patients randomized to receive rhATIII or placebo was well balanced. Of the 493 patients who were originally screened, 183 passed the initial screening test and had HDR (Medtronics) slopes of less than 80 seconds. Sixty-six of these patients $(66 / 493$ [13\%]) were found to be heparin resistant (ACT $<480$ seconds after $400 \mathrm{U} / \mathrm{kg}$ heparin), and 52 were randomly assigned to receive rhATIII $(\mathrm{n}=28)$ or placebo $(n=24)$. Patient characteristics, including age, sex, race, and weight, did not differ between groups.

In the intraoperative period, during which FFP administration was guided by study protocol, 6 (21\%) of 28 of patients in the rhATIII group received FFP compared with $22(92 \%)$ of 24 patients in the placebo group $(P<.001)$. This primary end point was similarly distributed across study centers for both treatment groups. In the 24-hour postoperative period, blood and blood component administration was guided by clinician discretion. There was no significant difference in the FFP, packed red blood cell, or platelet administration between the 2 groups in the 24-hour postoperative period. There was no significant difference between the groups in 24-hour postoperative bleeding.

ATIII activity was low at baseline in both groups and not significantly different in the 2 groups. In the rhATIII group, ATIII activity increased to within the normal range during $\mathrm{CPB}$. In the placebo group, ATIII activity decreased even lower than it had been at baseline during CPB (Figure 1).

There was a significant difference between the 2 groups in D-dimer concentration toward the end of CPB $(P<.05)$, with patients in the rhATIII group showing less evidence of fibrinolysis (ie, lower D-dimer levels). There was no significant difference between the 2 groups at any time point in fibrin monomer concentration or in the change in fibrin monomer concentration from baseline. The prothrombin fragment 1.2 concentrations increased significantly from baseline $(1.1 \pm 0.5 \mathrm{nmol} / \mathrm{L})$ in the placebo group at both time points during $\mathrm{CPB}(2.2 \pm 2.0$ and $3.9 \pm 2.8 \mathrm{nmol} / \mathrm{L}$, $P<.01)$. In the rhATIII group, there was no significant change in the prothrombin fragment 1.2 concentrations. The thrombin-antithrombin concentrations increased significantly in both groups during CPB $(P<.001)$, and this was more marked in the placebo group (Figure 2). 
rhATIII without FFP

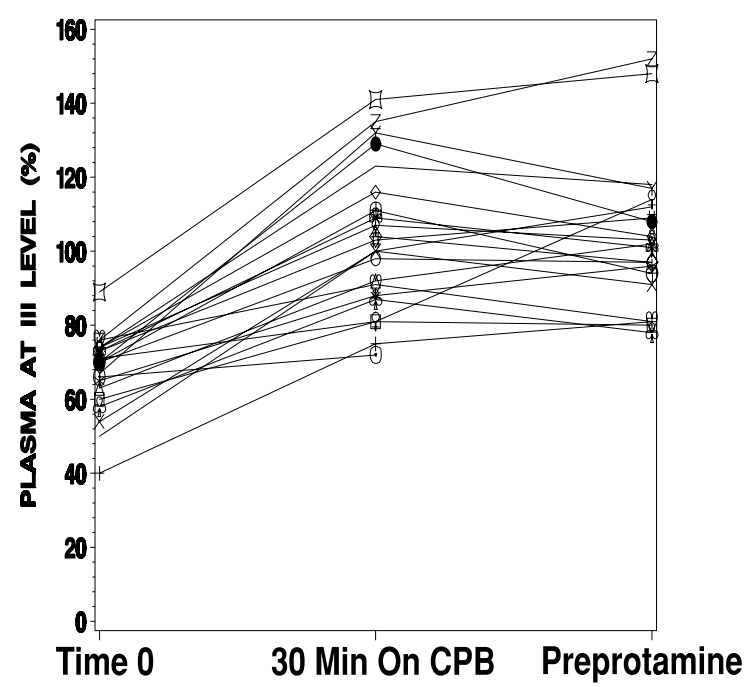

Placebo with FFP

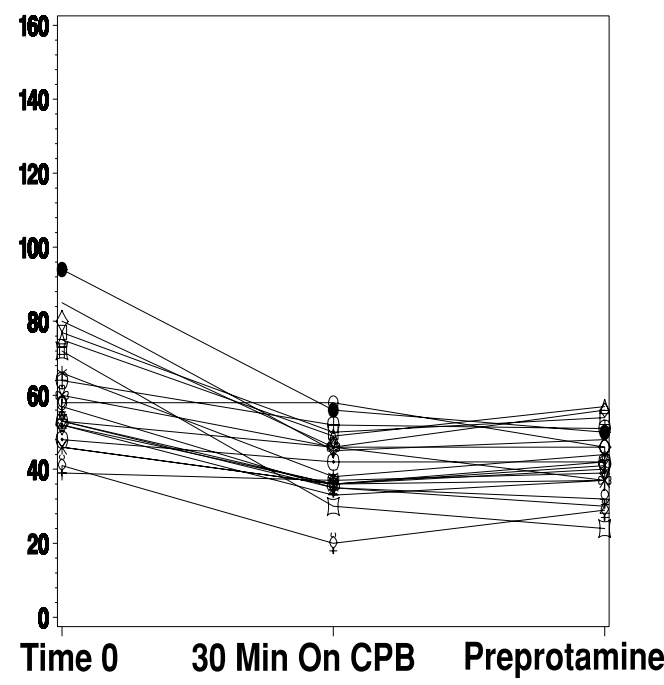

Figure 1. rhATIII activity for patients in each group at 3 time points around the CPB period. Time 0, Before institution of CPB; 30 min on CPB, 30 minutes after institution of $C P B$; Preprotamine, CPB period just before protamine administration. The normal range for plasma AT activity is $70 \%$ to $130 \%$.

rhATIII without FFP

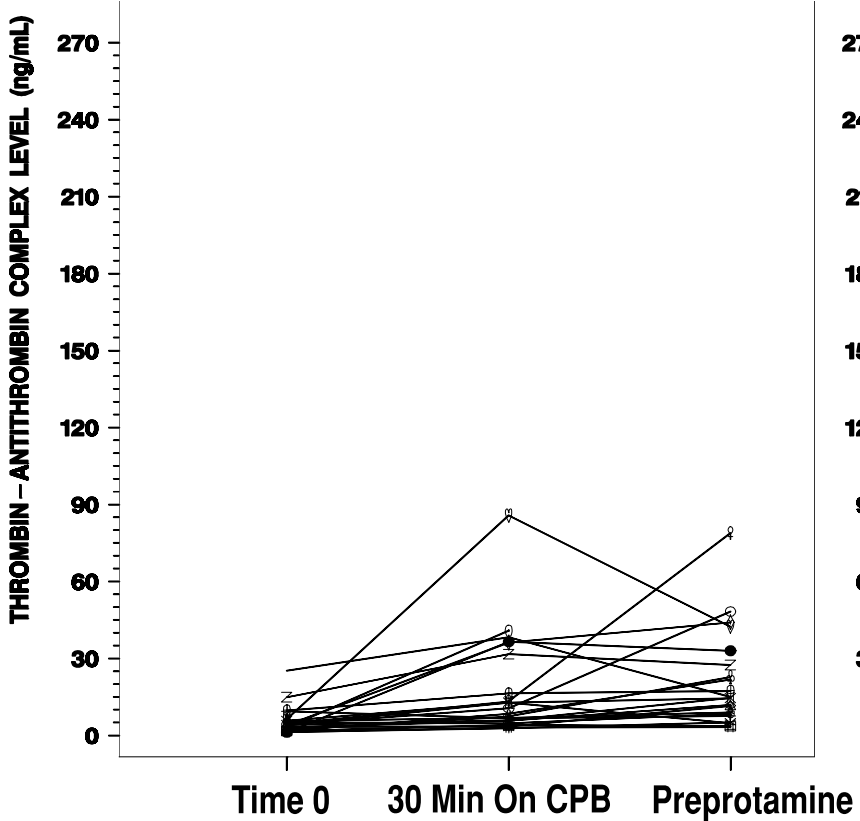

\section{Placebo with FFP}

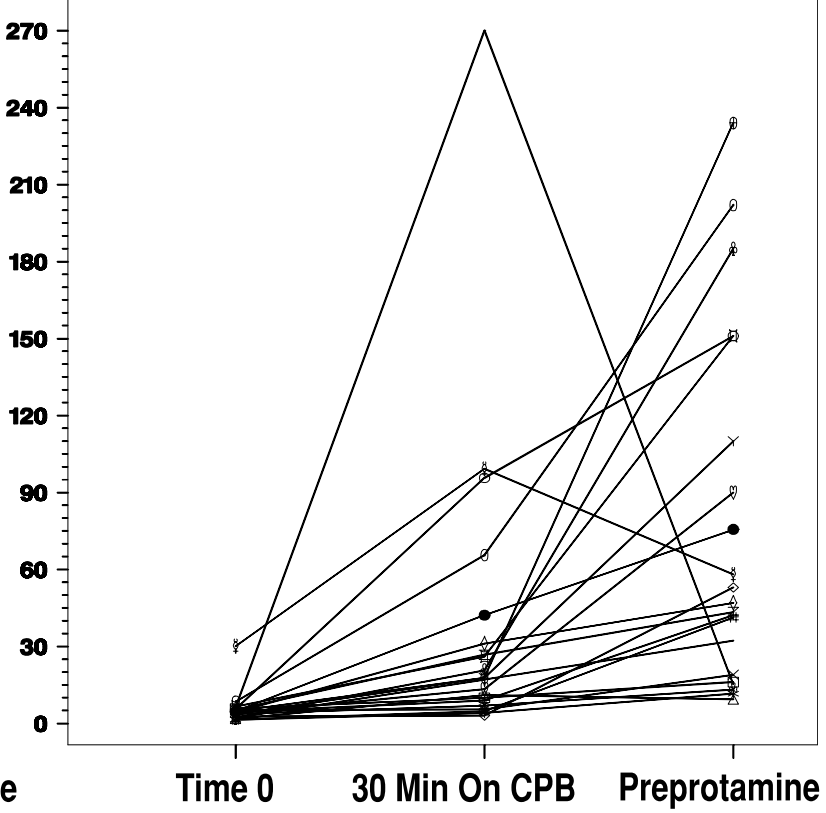

Figure 2. Thrombin-antithrombin complex concentrations for patients in each group at 3 time points around the CPB period. Time 0, Before institution of CPB; 30 min on CPB, 30 minutes after institution of CPB, Preprotamine, CPB period just before protamine administration. 
rhATIII without FFP

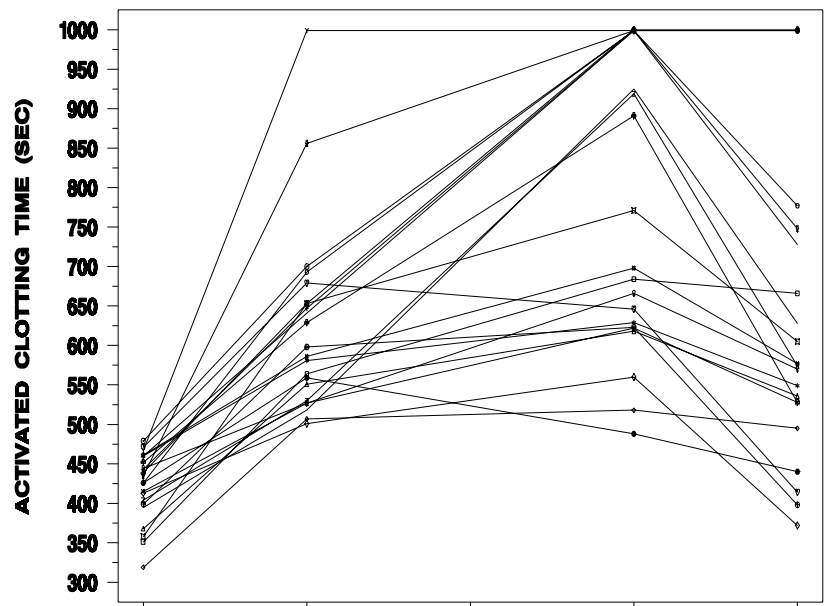

Placebo with FFP

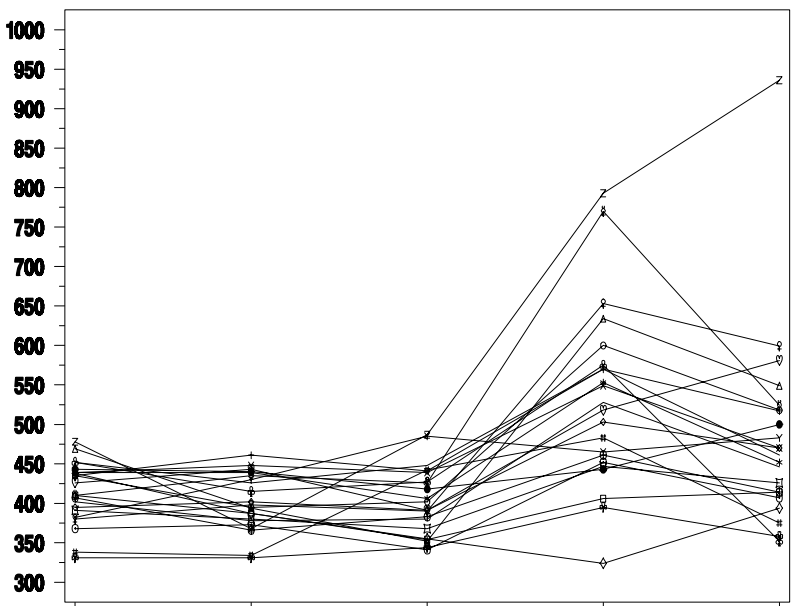

\section{Min
on CPB}

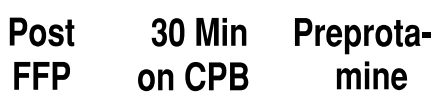

TX

Figure 3. Activated clotting times for patients in each group over the course of the study before and during CPB.

A total of $18(64 \%)$ of 28 patients treated with rhATIII required at least one additional heparin dose some time between administration of study medication and heparin reversal with protamine. Almost all of the placebo-treated patients (23/24 [96\%]) required at least one additional dose of heparin during the same period. Thirteen placebo-treated patients and 17 rhATIII-treated patients required only one additional dose of heparin. Ten placebo-treated patients and only 1 rhATIII-treated patient required between 2 and 4 additional doses of heparin.

The ACT was significantly increased in the rhATIII group compared with that in the placebo group after rhATIII administration and during CPB $(P<.001$, Figure 3$)$.

There were no patient deaths among the 28 patients randomized to be treated with rhATIII. The difference between treatment groups in the incidence of patients experiencing safety-related events (eg, volume of chest tube drainage, incidence of red blood cell transfusion, platelet transfusion, additional blood product transfusion, duration of surgical intensive care unit stay, duration of hospitalization, and 30-day incidence of rehospitalization, myocardial infarction, stroke, or death) was assessed. There was no significant difference in any of the major or minor adverse events reported between the treatment and placebo groups. The mean postoperative bleeding in the placebo group was $83 \mathrm{~mL} / \mathrm{h}$ compared with $82 \mathrm{~mL} / \mathrm{h}$ in the rhATIII group.

\section{Discussion}

The most important implication of this study is that it demonstrates that rhATIII effectively restores heparin responsiveness before CPB in the majority of heparin-resistant patients without increasing the incidence of bleeding. The primary end point of this randomized, double-blind, placebo-controlled study was achieved. The proportion of patients requiring administration of 2 units of FFP with the intention of achieving therapeutic anticoagulation, defined by an ACT of 480 seconds or longer, was significantly less $(P<.001)$ in the rhATIII-treated group $(21 \%)$ than in the placebo group $(92 \%)$. There were 6 patients who received rhATIII and FFP after heparin (400 U/kg) administration. This suggests that some patients $(21 \%)$ in the rhATIII group had a heparin resistance mechanism other than ATIII deficiency. ${ }^{9-12}$

The concept that ATIII deficiency is an important cause of heparin resistance in patients undergoing cardiac surgery was supported by the study data. Patients in the placebo group had a mean baseline ATIII activity of $61 \%$ (normal, 70\%-130\%). Furthermore, CPB-associated hemodilution induced a further decrease in ATIII activity to a mean of $42 \%$ activity in placebo-treated patients. In this group ATIII activity remained abnormally low throughout CPB. Importantly, despite the additional heparin the patients in the placebo group received, the ACTs remained low during $\mathrm{CPB}$ compared with those in patients in the rhATIII group 
(Figure 3). Administering additional heparin is not without potential complications, including increased bleeding and heparin rebound in the postoperative period. Administration of $75 \mathrm{U} / \mathrm{kg}$ rhATIII restored the mean plasma ATIII activity to greater than $100 \%$ activity despite hemodilution during CPB and maintained mean ATIII activity within normal physiologic range throughout $\mathrm{CPB}$. This finding supports the chosen dose of rhATIII ( $75 \mathrm{U} / \mathrm{kg})$. The large disparity in maximum ATIII plasma activity levels between the 2 groups demonstrates that administration of 2 units of FFP is not effective for normalizing plasma ATIII activity. Administration of rhATIII at a dose of $75 \mathrm{U} / \mathrm{kg}$ was well tolerated by heparin-resistant patients undergoing CPB. There was no difference in the incidence of serious adverse events or the incidence of clinically significant abnormal laboratory parameters between the 2 treatment groups. Importantly, rhATIII administration in this setting was not associated with increased intraoperative or postoperative blood loss. FFP was administered empirically to patients who were bleeding postoperatively, including to patients who had received rhATIII. This approach, however, has fallen out of favor on the basis of studies that demonstrate that prophylactic FFP $^{13}$ and platelet ${ }^{14}$ administration do not decrease bleeding. Postoperative transfusion of blood and blood components was similar in the 2 groups.

During CPB, blood and the artificial surface of the bypass circuit come into contact, providing a powerful stimulus to activate the hemostatic system. Subsequently, tissue factor and tissue plasminogen activator are retransfused to the patient through cardiotomy suction, resulting in systemic activation of the hemostatic system. ${ }^{2}$ Without anticoagulation, clots can form in the bypass circuit and might result in circuit occlusion or intracardiac thrombosis. ${ }^{15}$ In addition, with subtherapeutic anticoagulation, hemostatic system activation through thrombin and plasmin results in depletion of factors and platelets because of inadequate suppression of a disseminated intravascular coagulationlike consumptive state. To prevent these events, heparin is used to promote the action of ATIII in the blood, thereby reducing thrombin generation, thrombin activity, and fibrin generation extracorporeally and systemically. Therefore, heparin resistance might lead to inadequate anticoagulation during CPB and potentially lead to an increase in bleeding, thrombotic complications, or both.

Although gross clot formation in the extracorporeal circuit rarely occurs, activation of the hemostatic system still has important clinical implications. CPB activation of the hemostatic system has been implicated in the generation of cerebral microemboli, one of the proposed causes of stroke, and neurologic deficit often observed after CPB. ${ }^{16}$ Similarly, intraoperative coronary, ${ }^{17}$ pulmonary intracardiac, ${ }^{18}$ and $\mathrm{CPB}$ circuit thromboses have all been described during cardiac surgery. Postoperative bleeding might result from platelet and labile coagulation factor consumption caused by thrombin activity during $\mathrm{CPB} .^{2}$

Judging from surrogate markers, the results from this study are strongly suggestive that there was less hemostatic system activation in the group that received rhATIII. There was less activation of markers of both thrombin (eg, prothrombin fragments 1.2 and thrombin antithrombin complexes) and fibrinolytic activity (eg, D-dimer). Heparinresistant patients might be at risk for the consequences of inadequate anticoagulation during CPB. Previous studies have demonstrated that more pronounced anticoagulation with heparin can reduce transfusion requirements ${ }^{19}$ and preserve the hemostatic system. ${ }^{20}$ Studies have demonstrated that ATIII supplementation can attenuate thrombin and fibrinolytic activity. ${ }^{7}$ This suppression of thrombin and fibrinolytic activity, both of which usually increase during $\mathrm{CPB}$, might represent better inhibition of hemostatic activation than that achieved with heparin alone. ${ }^{7}$ The problem with detecting rare clinical events is that large numbers of patients are needed to detect these. For example, if giving rhATIII would result in a decrease in thrombotic complications in heparin-resistant patients from $10 \%$ to $5 \%, 433$ patients would be required in each group (power, $80 \% ; P=$ .05 ) to detect this. Assuming a $13 \%$ incidence of heparin resistance, 6662 patients $(866 / 6662$ [13\%]) would have to be recruited to such a study. It is for this reason that we looked at surrogate markers.

This is the second randomized, double-blind, placebocontrolled trial that has demonstrated that rhATIII is effective in the management of heparin resistance in patients undergoing cardiac surgery with $\mathrm{CPB} .{ }^{21}$ There was a suggestion in the other study that rhATIII might be associated with increased postoperative bleeding. ${ }^{21}$ There was no increase in postoperative bleeding with rhATIII in the present study. Heparin resistance was caused by ATIII deficiency in the majority of cases because the ACT was prolonged to the therapeutic range ( $>480$ seconds) in $79 \%$ of patients after rhATIII administration. Administration of $75 \mathrm{U} / \mathrm{kg}$ rhATIII restored ATIII activity to within the normal range, which led to improved heparin responsiveness, adequate anticoagulation for $\mathrm{CPB}$, and avoidance of FFP administration in the majority of cases. In contrast, ATIII activity decreased further from baseline in placebo-treated patients and required FFP treatment and additional heparin administration to obtain adequate anticoagulation before CPB initiation. Two units of FFP did not restore ATIII activity to the normal range.

In summary, the present study demonstrated that $75 \mathrm{U} / \mathrm{kg}$ rhATIII can be administered to heparin-resistant patients undergoing CPB and effectively restores heparin responsiveness in the vast majority of cases. There was strong evidence that rhATIII administration decreased hemostatic system activation during $\mathrm{CPB}$. In addition, this prospective 
trial demonstrates that rhATIII administration for management of heparin resistance might substantially reduce exposure to FFP.

We thank the following individuals who participated in this study: Scott Streckenbach, MD, A. Hoeft, MD, L. D. J. Booij, MD, J. Streisand, and D. Meeker, MD.

\section{References}

1. Young JA, Kisker CT, Doty DB. Adequate anticoagulation during cardiopulmonary bypass determined by activated clotting time and the appearance of fibrin monomer. Ann Thorac Surg. 1978;26:231-40.

2. Despotis GJ, Gravlee G, Filos K, Levy J. Anticoagulation monitoring during cardiac surgery: a review of current and emerging techniques. Anesthesiology. 1999;91:1122-51.

3. Esposito RA, Culliford AT, Colvin SB, Thomas SJ, Lackner H, Spencer FC. Heparin resistance during cardiopulmonary bypass. The role of heparin pretreatment. J Thorac Cardiovasc Surg. 1983;85:34653.

4. Anderson EF. Heparin resistance prior to cardiopulmonary bypass. Anesthesiology. 1986;64:504-7.

5. Despotis GJ, Levine V, Joist JH, Joiner-Maier D, Spitznagel E. Antithrombin III during cardiac surgery: effect on response of activated clotting time to heparin and relationship to markers of hemostatic activation. Anesth Analg. 1997;85:498-506.

6. Lemmer JH Jr, Despotis GJ. Antithrombin III concentrate to treat heparin resistance in patients undergoing cardiac surgery. $J$ Thorac Cardiovasc Surg. 2002;123:213-7.

7. Levy JH, Despotis GJ, Szlam F, Olson P, Meeker D, Weisinger A. Recombinant human transgenic antithrombin in cardiac surgery: a dose-finding study. Anesthesiology. 2002;96:1095-102.

8. Sabbagh AH, Chung GK, Shuttleworth P, Applegate BJ, Gabrhel W. Fresh frozen plasma: a solution to heparin resistance during cardiopulmonary bypass. Ann Thorac Surg. 1984;37:466-8.

9. Shanberge JN, Quattrociocchi-Longe TM, Martens MH. Interrelationship of protamine and platelet factor 4 in the neutralization of heparin. Thromb Res. 1987;46:89-100.

10. Vuylsteke A, Mills RJ, Crosbie AE, Burns TI, Latimer RD. Increased pre-operative platelet counts are a possible predictor for reduced sensitivity to heparin. Br J Anaesth. 2000;85:896-8.
11. Hirsh J, Anand SS, Halperin JL, Fuster V. Guide to anticoagulant therapy: Heparin: a statement for healthcare professionals from the American Heart Association. Circulation. 2001;103:2994-3018.

12. Teoh KH, Young E, Bradley CA, Hirsh J. Heparin binding proteins. Contribution to heparin rebound after cardiopulmonary bypass. Circulation. 1993;88(suppl II):II420-5.

13. Consten EC, Henny CP, Eijsman L, Dongelmans DA, van Oers MH. The routine use of fresh frozen plasma in operations with cardiopulmonary bypass is not justified. J Thorac Cardiovasc Surg. 1996;112: $162-7$.

14. Reed RL 2nd, Ciavarella D, Heimbach DM, Baron L, Pavlin E, Counts $\mathrm{RB}$, et al. Prophylactic platelet administration during massive transfusion. A prospective, randomized, double-blind clinical study. Ann Surg. 1986;203:40-8.

15. Fanashawe MP, Shore-Lesserson L, Reich DL. Two cases of fatal thrombosis after aminocaproic acid therapy and deep hypothermic circulatory arrest. Anesthesiology. 2001;95:1525-7.

16. Stump DA, Rogers AT, Hammon JW, Newman SP. Cerebral emboli and cognitive outcome after cardiac surgery. J Cardiothorac Vasc Anesth. 1996;10:113-9.

17. Loubser PG, McCarthy JJ, Attar M. Coronary thrombosis associated with antithrombin-III deficiency. J Cardiothorac Vasc Anesth. 1997; 11:756-9.

18. Cheung AT, Levin SK, Weiss SJ, Acker MA, Stenach N. Intracardiac thrombus: a risk of incomplete anticoagulation for cardiac operations. Ann Thorac Surg. 1994;58:541-2.

19. Despotis GJ, Joist JH, Hogue CW Jr, Alsoufiev A, Kater K, Goodnough LT, et al. The impact of heparin concentration and activated clotting time monitoring on blood conservation: a prospective, randomized evaluation in patients undergoing cardiac operation. J Thorac Cardiovasc Surg. 1995;110:46-54.

20. Despotis GJ, Joist JH, Hogue CW Jr, Alsoufiev A, Joiner-Maier D, Santoro SA, et al. More effective suppression of hemostatic system activation in patients undergoing cardiac surgery by heparin dosing based on heparin blood concentrations rather than ACT. Thromb Haemost. 1996;76:902-8.

21. Avidan MS, Levy JH, Scholz J, Delphin E, Rosseel PM, Howie MB, et al. A phase III, double-blind, placebo-controlled, multicenter study on the efficacy of recombinant human antithrombin in heparin-resistant patients scheduled to undergo cardiac surgery necessitating cardiopulmonary bypass. Anesthesiology. 2005;102:276-84. 